\title{
\#getintouch! Eine vergleichende Analyse der digitalen Points of Contact in Zürcher Gemeinden
}

\author{
Damaris Fischer (D) - Alexander Mertes $•$ Selina Hänni
}

Eingegangen: 11. März 2021 / Angenommen: 24. Juni 2021 / Online publiziert: 20. Juli 2021

(C) Der/die Autor(en) 2021

Zusammenfassung Die digitale Transformation der Verwaltung in der Schweiz schreitet voran. Ein zentraler Aspekt der digitalen Verwaltung ist der Zugang zu Dienstleistungen über das Internet. Im Zentrum stehen dabei oftmals die Behördenwebseiten, da verschiedene Anspruchsgruppen darüber Informationen und Dienstleistungen beziehen. Demzufolge lösen die Webseiten in einer digitalen Verwaltung den Schalter als primären Point of Contact ab. Während die Privatwirtschaft den Mehrwert digitaler Points of Contact bereits erkannt hat, ist der Umsetzungsstand in der Verwaltung heute nicht bekannt. Dieser Beitrag erhebt das Angebot digitaler Kontaktmöglichkeiten in allen Zürcher Gemeinden. Es zeigt sich, dass klassische digitale Points of Contact wie eine E-Mail-Adresse oder ein Online-Schalter von der Mehrheit der Gemeinden angeboten wird, während innovativere Formate wie ein Stadtmelder oder ein Chatbot seltener anzutreffen sind. Die Grösse der Gemeinde scheint zudem einen positiven Einfluss auf die Vielfalt des Angebotes von digitalen Points of Contact zu haben. Der Beitrag stellt eine erstmalige Übersicht zum Stand digitaler Kontaktmöglichkeiten in den Zürcher Gemeinden dar.

Schlüsselwörter Digitale Transformation · Digitale Verwaltung · E-Government · Behördenwebseiten · Points of Contact 


\section{\#getintouch! A comparative analysis of digital points of contact in Zurich municipalities}

Abstract The digital transformation of the administration is progressing in Switzerland. A central aspect of digital administration is the access to services via the internet. The websites of the authorities are often at the centre of this, as various stakeholder groups obtain information and services this way. Consequently, the websites in a digital administration replace the counter as the primary point of contact. While the private sector has already recognised the added value of digital points of contact, the status of implementation in the public sector is not known today. This article shines light on the offer of digital points of contact in all Zurich municipalities. It shows that classic digital points of contact such as an email address or an online counter are offered by the majority of the municipalities, while more innovative formats such as a city messenger or a chatbot are less common. The size of the municipality also seems to have a positive influence on the variety of digital points of contact offered. The paper presents a first overview of the state of digital points of contact in Zurich municipalities.

Keywords Digital Transformation - Digital Administration · E-Government · Public Administration Websites · Points of Contact

\section{Einführung}

Die Bevölkerung gewöhnt sich dank innovativen und disruptiven Unternehmen, wie beispielsweise Amazon, Netflix oder Zoom, zunehmend an digitale Dienstleistungen, was die Entwicklung neuer digitaler Angebote beschleunigt (European Commission Joint Research Centre 2020). Diese Entwicklungen lösen Erwartungen an die Verwaltung aus, ebenfalls digitale Dienstleistungen anzubieten (Brüesch et al. 2017; Mertes et al. 2020; Pleger et al. 2020). Um diesem Bedürfnis nachzugehen, hat sich das Angebot an digitalen Services der Behörden in den letzten Jahren vergrössert, beispielsweise im Bereich der Partizipation, der Bildung oder der Verwaltungsleistungen im Allgemeinen (Cahlikova und Bundi 2020; Fischer et al. 2020a; Teucher und Lang 2020).

Ein zentraler Aspekt der digitalen Transformation der Verwaltung ist das Angebot Dienstleistungen über das Internet. Dieser Einsatz von Informations- und Kommunikationstechnologien, um die Zusammenarbeit zwischen Wirtschaft, Gesellschaft und Verwaltung zu erleichtern, wird mit dem Begriff E-Government erfasst (Neuroni et al. 2019). Die Entwicklung hin zu einer digitalen Verwaltung führt zu einer Veränderung der Interaktion zwischen Wirtschaft und Gesellschaft mit der öffentlichen Verwaltung, es wird eine neue Beziehung auf digitaler Ebene kreiert. Im Zentrum stehen dabei oftmals die Behördenwebseiten, da verschiedene Anspruchsgruppen darüber Informationen und Dienstleistungen beziehen. Demzufolge könnten die Webseiten, über welche beispielsweise auf das Bürger*innenkonto zum Bezug verschiedener Dienstleistungen zugegriffen werden kann, in einer digitalen Verwaltung den klassischen Schalter als primären Point of Contact ablösen (Wong und 
Welch 2004). Auf den Webseiten können Chatbots, Stadtmelder oder andere Formate angeboten oder verknüpft werden, um für die Anspruchsgruppen der Verwaltung verschiedene digitale Kontaktmöglichkeiten zu schaffen. Während die Privatwirtschaft den Mehrwert digitaler Points of Contact wie erhöhte Effizienz oder Automatisierungspotentiale bereits erkannt hat, ist der Umsetzungsstand in der Verwaltung heute nicht bekannt.

Diese Untersuchung stellt sich in diesem Kontext die Frage, wie weitgehend Schweizer Gemeinden bereits digitale Kontaktmöglichkeiten anbieten. Als Untersuchungsgegenstand dient der Kanton Zürich mit 162 Gemeinden. Die Forschungsfrage lautet: Welche digitalen Kontaktmöglichkeiten bestehen in den Zürcher Gemeinden und inwiefern hängt das Angebot mit der Grösse der Gemeinde zusammen? Um diese Frage zu beantworten, wurden die digitalen Kontaktmöglichkeiten auf den Webseiten der 162 Zürcher Gemeinden quantitativ erhoben. Zudem wurde untersucht, inwiefern die Grösse stellvertretend für die Ressourcen einer Gemeinde einen Einfluss auf die Vielfalt des Angebotes an digitalen Points of Contact hat.

Die Studie gliedert sich in die folgenden Abschnitte: Nach der Einführung wird der Stand der Forschung dargelegt. Dabei wird insbesondere auf die Entwicklung der digitalen Transformation auf den drei Schweizer Staatsebenen Bund, Kantone und Gemeinden eingegangen. Es folgt eine Übersicht zu den Methoden und Daten und anschliessend werden die Resultate präsentiert. Im letzten Abschnitt werden die Ergebnisse diskutiert und es wird ein Fazit gezogen.

\section{Theoretischer Rahmen}

Die öffentliche Verwaltung hat in den letzten Jahrzehnten verschiedene Reformen durchlaufen: Das Bürokratiemodell nach Weber (siehe Weber 1980) wurde durch das Aufkommen des New Public Managements in den 1990er-Jahren verdrängt. Diese Verwaltungsreform veränderte die Rolle der Verwaltung vom starren Verwaltungsapparat zur heutigen Dienstleistungsorientierung (Schedler und Proeller 2011). Die Ansätze des New Public Managements sind jedoch stark nach innen gerichtet und beschreiben die Rolle der Bürger*innen als passive Kund*innen. Dies führte zu Kritik, denn heute wird von der Verwaltung vermehrt gefordert, sich nach aussen zu öffnen und Anspruchsgruppen in die Entscheidungsprozesse stärker zu integrieren (Brüesch et al. 2017). Diese Entwicklung hin zu einem partnerschaftlichen Verhältnis zwischen Verwaltung und Anspruchsgruppen wird unter dem Begriff Public Governance diskutiert. Im Gegensatz zu den Ansätzen des New Public Managements werden Bürger*innen unter dem Public Governance nicht bloss als Leistungsempfänger*innen oder Kund*innen betrachtet, sondern werden als Mitgestalter*innen stärker in die Entwicklung und Entscheidungsfindung der Verwaltung einbezogen (Brüesch et al. 2017). Die Entwicklung zu einem kooperativen Staat wird durch einen weiteren Trend unterstützt: Die Digitalisierung schafft neue Möglichkeiten für die Verwaltung, ihre Dienstleistungen kundenorientiert zu gestalten und anzubieten und sich dadurch zu einer digitalen Verwaltung zu entwickeln (Dietrich et al. 2018; Fischer et al. 2020b). Ebenfalls wird im Diskurs um die digitale Verwaltung oftmals der Begriff E-Government genannt (Brüesch et al. 2017; Mertes et al. 2020; 
Neuroni et al. 2019). Durch die Schaffung von E-Government-Angeboten rückt der analoge Schalter vermehrt in den Hintergrund und schafft Platz für digitale Kontaktmöglichkeiten mit der Gemeinde. Für die vorliegende Studie werden die digitalen Points of Contact als Bestandteil des E-Governments gesehen. Im Folgenden wird genauer auf die Chancen und das Potenzial des E-Governments und dessen Umsetzungsstand auf allen drei Staatsebenen eingegangen.

\subsection{E-Government in der digitalen Verwaltung}

E-Government kann breit gefasst als der „Einsatz von IT im öffentlichen Sektor und den damit verbundenen Wandel“" (Neuroni et al. 2019, S. 164-165) umschrieben werden. Das Ziel des E-Governments ist unter anderem, öffentliche Dienstleistungen für Wirtschaft und Bevölkerung auf digitalem Wege zu erbringen, wobei diese keine zusätzlichen Kosten verursachen sollen (Brüesch et al. 2017, 2020; Neuroni et al. 2019; Wong und Welch 2004). Im Zentrum des E-Governments stehen dabei oftmals die Behördenwebseiten, da verschiedene Anspruchsgruppen darüber Informationen und Dienstleistungen beziehen. Demzufolge lösen die Webseiten in einer digitalen Verwaltung den Schalter als primären Point of Contact ab (Wong und Welch 2004).

Um digitale Kontaktmöglichkeiten mit der Verwaltung wahrzunehmen, muss die Bevölkerung in der Lage sein, auf diese zuzugreifen. Die Schweizer Bevölkerung ist gemäss dem Global Information Technology Report des Weltwirtschaftsforums ausgerüstet, um von den Chancen eines E-Governments, im Besonderen der digitalen Kontaktmöglichkeiten, profitieren zu können: Die Schweiz verfügt nebst guter Infrastruktur im Allgemeinen auch über sehr gut ausgebildete Arbeitskräfte (Baller et al. 2016). Ferner benutzen $92 \%$ der Schweizer Bevölkerung das Internet, gut $47 \%$ verfügen über einen festen Breitbandanschluss und auf 100 Einwohner*innen kommen 130 Mobilabonnemente (Statista 2019). In Bezug auf den Entwicklungsstand des E-Governments in der Schweiz zeigt sich, dass das Potenzial trotz dieser guten Grundlage noch nicht ausgeschöpft ist: Im E-Government Survey 2020 der United Nations belegt die Schweiz den 16. Platz unter den 193 untersuchten Staaten (United Nations 2020). Dieses Ergebnis bedeutet im Vergleich zur letzten Erhebung 2018 einen Verlust eines Ranges trotz höher Bewertung. Dies lässt darauf schliessen, dass sich andere Staaten in den letzten Jahren im Bereich des E-Governments stärker entwickelt haben (siehe dazu auch Neuroni et al. 2019).

\subsection{Umsetzung des E-Governments in der Schweiz}

Seit 2008 verfolgen die drei Staatsebenen Bund, Kantone und Gemeinden in der Schweiz eine gemeinsame E-Government Strategie, welche die Entwicklungen im Bereich der digitalen Transformation vereinheitlichen sollten. Im Folgenden soll kurz aufgezeigt werden, welche Schwerpunkte die aktuelle E-Government Strategie setzt und wie es um die Digitalisierung der Gemeinden in der Schweiz steht.

Auf Bundesebene wurde die E-Government Strategie 2020-2023 verabschiedet, um die digitale Transformation in der Schweiz weiter voranzutreiben und ein E-Government zu etablieren. Ziel dieser Strategie ist die Förderung digital angebotener Dienstleistungen auf allen drei Staatsebenen gemäss dem Prinzip digital first. Damit 
ist gemeint, dass Behörden ihre digitalen Dienstleistungen so benutzerfreundlich gestalten, dass neben dem Erhalt der analogen Kanäle die digitalen von den Anspruchsgruppen bevorzugt werden. Weiter definiert die E-Government Strategie 2020-2023 vier Handlungsfelder, auf welche sich die Aktivitäten der Schweiz im Bereich des E-Governments fokussieren. Die Handlungsfelder für die Jahre 2020-2023 sind nachfolgend kurz erläutert, wobei insbesondere das Handlungsfeld Interaktion und Partizipation für den vorliegenden Beitrag bedeutsam ist (E-Government Schweiz 2019):

Interaktion und Partizipation Bund, Kantone und Gemeinden fördern den elektronischen Austausch mit ihren Anspruchsgruppen und bieten elektronische Mitwirkungsmöglichkeiten an.

Basisdienste und Infrastruktur Bund, Kantone und Gemeinden legen den Fokus auf gemeinsame Aktivitäten, die in der E-Government Strategie bestimmt sind und fördern offene Schnittstellen sowie den Aufbau gemeinsamer Dienste. E-Government Schweiz identifiziert Leistungen, die entweder zentral vom Bund oder gemeinsam erbracht werden.

Organisation und rechtliche Grundlagen Bund, Kantone und Gemeinden definieren die verbindlichen Zuständigkeiten in der Zusammenarbeit im E-Government, legen ihre Ressourcen in den relevanten Bereichen zusammen und harmonisieren rechtliche Grundlagen.

Vertrauen und Wissen Bund, Kantone und Gemeinden setzen sich für den notwendigen Kulturwandel und Wissensaufbau innerhalb der Verwaltung sowie die Vertrauensschaffung in der Bevölkerung ein.

Nebst der E-Government Strategie 2020-2023 wurden gleichzeitig auch die öffentlich-rechtliche Rahmenvereinbarung und der Umsetzungsplan E-Government Schweiz verabschiedet. Während diese Vereinbarung insbesondere die Organisation sowie das Budget für die Umsetzung der E-Government Strategie 2020-2023 beinhaltet, regelt der Umsetzungsplan die gemeinsamen Aktivitäten aller drei Staatsebenen und definiert die Ziele und Massnahmen zur Umsetzung der E-Government Strategie 2020-2023 (E-Government Schweiz 2019). Die E-Government Strategie 2020-2023 sieht vor, dass sich Bund, Kantone und Gemeinden gemeinsam auf den Weg zur digitalen Verwaltung machen. Es stellt sich nun die Frage, wie weit die zweite und dritte Staatsebene (Kantone und Gemeinden) bereits fortgeschritten sind und inwiefern sich Unterschiede in der Entwicklung festmachen lassen.

Bezüglich des Digitalisierungsstandes der Gemeinden sind bisher wenige Studien vorhanden. In der nationalen E-Government Studie der Schweiz werden über 1000 Gemeinden zu ihrem aktuellen digitalen Angebot befragt. Dabei zeigt sich, dass die Mehrheit der Services zum Erhebungszeitpunkt analog angeboten wird. Die Anmeldung in der Wohngemeinde kann am häufigsten elektronisch durchgeführt werden (in $21 \%$ der befragten Gemeinden, vor allem in Deutschschweizer Gemeinden), die Einbürgerung kann mit ein paar Ausnahmen nur analog vorgenommen werden (Buess et al. 2019). Als erschwerende Faktoren für die Digitalisierung bestimm- 
ter Services nennen die Gemeinden am häufigsten Personalressourcen, Zeitaufwand und Budgetrestriktionen (Buess et al. 2019). Die Befunde zeigen, dass die digitale Transformation in den Gemeinden erst am Anfang steht und noch viel Potenzial im Bereich des Angebots von digitalen Dienstleistungen vorhanden ist.

\subsection{Die Bedeutung digitaler Kontaktmöglichkeiten}

Um den Weg zum E-Government zu ebnen, müssen Gemeinde gemäss dem Handlungsfeld Interaktion und Partizipation der E-Government Strategie 2020-2023 ihren verschiedenen Anspruchsgruppen ermöglichen, Dienstleistungen online beziehen und über digitale Kanäle in Kontakt treten zu können. Dabei stellt oftmals die Webseite die zentrale Anlaufstelle für verschiedene Anliegen dar, wodurch der analoge Schalter an Bedeutung verliert (Wong und Welch 2004). Es wird kurz aufgezeigt, welche Studien zum aktuellen Stand der angebotenen digitalen Points of Contact in den Schweizer Gemeinden bereits bestehen und zu welchen Ergebnissen diese kommen.

Eine Studie von C-Factor hat mit Hilfe einer Online-Befragung im Herbst 2019 in einer Zürcher Gemeinde die aktuellen und von der Bevölkerung gewünschten Kommunikationsmittel untersucht. Die Autor*innen kommen zum Schluss, dass die klassischen Kommunikationsmittel einer Gemeinde - wie das Mitteilungsblatt oder das Einwohner*innenschreiben - nicht mehr zur heutigen Zeit passen: $88 \%$ der Befragten wünschen sich vermehrt digitale Angebote. Insbesondere wird eine Gemeinde-App und der Einsatz von Sozialen Medien gefordert (C-Factor 2019). Diese Ergebnisse zeigen, dass sich digitale Points of Contact zumindest in der untersuchten Gemeinde noch nicht durchgesetzt haben, obwohl diese verschiedenen Vorteile wie eine Effizienzsteigerung oder ein Automatisierungspotential aufweisen. Gleichzeitig ermöglicht ein breites Angebot von verschiedenen Points of Contact einen stärkeren Einbezug der Bevölkerung in die Entscheidungsfindung von Gemeinden, was auch die Forderung der Entwicklung hin zu einer partnerschaftlichen Verwaltung unterstützt (vgl. Abschnitt 2).

In der Privatwirtschaft wurde das Potenzial von digitalen Kontaktmöglichkeiten bereits erkannt: Indem verschiedene Arten von Kontaktmöglichkeiten zielgruppenspezifisch angeboten werden, können Kund*innen stärker involviert und ihre Bedürfnisse besser abgeholt werden (Dahl et al. 2018). Zudem ermöglichen digitale Points of Contact auch den zeit- und ortsunabhängigen Kontakt zum Unternehmen, sodass bestimmte Dienstleistungen jederzeit zur Verfügung stehen (Straker et al. 2015). In einer Studie identifizieren Straker et al. (2015) die häufigsten digitalen Kontaktpunkte eines Unternehmens mit seinen Kund*innen. Diese sind die Webseite, Apps, E-Mail, und soziale Medien. Diese Kanäle werden von Unternehmen für unterschiedliche Zwecke eingesetzt, beispielsweise um Informationen bereit zu stellen, eine Beteiligungsmöglichkeit für Kund*innen zu schaffen oder um Feedback entgegenzunehmen (Straker et al. 2015).

Bis heute fehlen jedoch Analysen, welche digitalen Kontaktmöglichkeiten von den Gemeinden angeboten werden und inwiefern die Ressourcen bezüglich der Vielfältigkeit des Angebots eine Rolle spielen. Es wird vermutet, dass kleinere Gemeinden aufgrund eingeschränkter Ressourcen ein weniger vielfältiges Angebot an 
digitalen Kontaktmöglichkeiten anbieten (vgl. Abschnitt 2.2). Daraus lässt sich die folgende Hypothese ableiten:

- H1: Kleinere Gemeinden haben ein weniger vielfältiges Angebot an digitalen Points of Contact als grössere Gemeinden.

\section{Erhebungsdesign}

Um die Forschungsfrage zu beantworten, wurden die Gemeinden im Kanton Zürich untersucht. Dafür wurden im Oktober 2020 alle 162 Webseiten des Kantons Zürich besucht und die angebotenen Kontaktmöglichkeiten erhoben. Es wurden die folgenden Points of Contact auf den Webseiten beobachtet und folglich in die Analyse aufgenommen: Online-Schalter, Bürger*innen-/Benutzer*innen-Konto, Stadtmelder, Gemeinde-App, Newsletter, E-Mail, Chatbot und Soziale Medien. Andere Kontaktmöglichkeiten, wie beispielsweise die Telefonnummer oder Postanschrift wurden ausgeschlossen, da diese nicht zu den digitalen Points of Contact zählen. Des Weiteren wurden Kontaktmöglichkeiten, die nur vereinzelt auf den Webseiten der Gemeinden beobachtet wurden (bspw. Beschwerdekiste, Partizipationsplattformen wie Crossiety) ebenfalls für die Analyse ausgeschlossen. Die erhobenen Kontaktmöglichkeiten werden nachfolgend kurz beschrieben, wie sie in diesem Beitrag verstanden werden.

Online-Schalter Ein Online-Schalter ermöglicht es, allgemeine Dienstleistungen einer Gemeinde, beispielsweise die Bestellung einer Parkkarte oder die Meldung des Wegzuges online und somit unabhängig von den Öffnungszeiten der Gemeinde vorzunehmen.

Bürger*innen-/Benutzer*innenkonto Das Bürger*innenkonto beziehungsweise das Benutzer*innen-Konto ist meist eng mit dem Online-Schalter verknüpft. Im Unterschied zum Online-Schalter ermöglicht es den Einwohner*innen, personalisierte Dienstleistungen vorzunehmen wie beispielsweise eine Raumreservation in einem öffentlichen Gebäude. Des Weiteren können auch Services beansprucht werden, die nur den Einwohner*innen vorbehalten sind, zum Beispiel den Bezug einer Tageskarte für den Bahnverkehr oder die Bestellung eines Grüncontainers.

Stadtmelder Mit einem Stadtmelder können Personen Meldung bezüglich Schäden an der Infrastruktur in einer Gemeinde, wie etwa einer kaputten Straßenlaterne, direkt der Gemeinde melden.

Gemeinde-App Immer mehr Menschen nutzen das Internet über ihr Smartphone. Aus diesem Grund bieten Gemeinden für die Bevölkerung auch Apps an. So können die Informationen und Dienstleistungen, welche sie auf der Webseite zur Verfügung stellen, einfacher mit dem Smartphone abgerufen werden. 
Newsletter Verschiedene Gemeinden bieten das Abonnieren eines Newsletters auf ihrer Webseite an. Dadurch werden Interessierte über Aktualitäten und Veranstaltungen in der Gemeinde informiert.

E-Mail Ein weiterer digitaler Kontaktpunkt stellt die Korrespondenz über E-Mails dar. Geben die Gemeinden die E-Mail-Adressen ihrer Ämter und Mitarbeitenden an, kann die Bevölkerung direkt mit dem zuständigen Amt beziehungsweise den zuständigen Mitarbeitenden in Kontakt treten.

Chatbot Der Chatbot ist ein textbasiertes Dialogsystem, welches der Kommunikation mit Webseiten-Besucher*innen dient. Informationen oder Services können direkt über den Chatbot bezogen werden.

Soziale Medien Soziale Medien werden zunehmend auch von Gemeinden genutzt, um mit ihrer Bevölkerung auf informellen Wegen in Kontakt zu treten, sie zu informieren und auf Veranstaltungen hinzuweisen. Es wurden folgende Soziale Medien auf den Gemeindewebseiten beobachtet: Facebook, Instagram, Twitter und YouTube.

Diese Kontaktmöglichkeiten wurden binär mit den Kategorien „Ja“ und „Nein“ erhoben. Es wurde ein Gesamtscore berechnet, der die Werte zwischen 0 und 1 annehmen kann und aussagt, wie stark digitale Points of Contact in einer Gemeinde vorhanden sind. Bei den erhobenen Points of Contact, bei denen es mehrere Gestaltungsmöglichkeiten gibt, beispielsweise bei den Sozialen Medien, wurde der

Tab. 1 Übersicht Erhebung der Kontaktmöglichkeiten auf den Gemeindewebseiten

\begin{tabular}{|c|c|c|}
\hline Point of Contact & Frage & $\begin{array}{l}\text { Antwortkategorien } \\
\text { und Codierung }\end{array}$ \\
\hline Online-Schalter & Gibt es einen Online-Schalter? & $\begin{array}{l}\text { 1: Ja } \\
0: \text { Nein }\end{array}$ \\
\hline $\begin{array}{l}\text { Benutzer*innen-/ } \\
\text { Bürger*innenkonto }\end{array}$ & Gibt es ein Benutzerkonto/Bürgerkonto? & $\begin{array}{l}\text { 1: Ja } \\
0: \text { Nein }\end{array}$ \\
\hline Stadtmelder & Gibt es einen Stadtmelder? & $\begin{array}{l}\text { 1: Ja } \\
0: \text { Nein }\end{array}$ \\
\hline Gemeinde-App & Gibt es eine Gemeinde-App? & $\begin{array}{l}\text { 1: Ja } \\
0: \text { Nein }\end{array}$ \\
\hline Newsletter & $\begin{array}{l}\text { Ist es möglich, den Newsletter über die Startseite der } \\
\text { Webseite zu abonnieren? }\end{array}$ & $\begin{array}{l}\text { 1: Ja, Newsletter } \\
\text { kann auf der Startsei- } \\
\text { te abonniert werden } \\
0: \text { Nein }\end{array}$ \\
\hline E-Mail & $\begin{array}{l}\text { Ist die allgemeine E-Mail-Adresse auf der Webseite? } \\
\text { Ist die E-Mail-Adresse je Fachamt auf der Webseite? } \\
\text { Ist die E-Mail-Adresse je Mitarbeiter*in auf der Web- } \\
\text { seite? }\end{array}$ & $\begin{array}{l}\text { Mittelwert aus allen } \\
\text { Fragen mit } \\
\text { 1: Ja } \\
0: \text { Nein }\end{array}$ \\
\hline Chatbot & Gibt es einen Chatbot? & $\begin{array}{l}\text { 1: Ja } \\
0: \text { Nein }\end{array}$ \\
\hline Soziale Medien & $\begin{array}{l}\text { Gibt es Verlinkungen zu Facebook auf der Webseite? } \\
\text { Gibt es Verlinkungen zu Instagram auf der Webseite? } \\
\text { Gibt es Verlinkungen zu Twitter auf der Webseite? } \\
\text { Gibt es Verlinkungen zu YouTube auf der Webseite? }\end{array}$ & $\begin{array}{l}\text { Mittelwert aus allen } \\
\text { Fragen mit } \\
\text { 1: Ja } \\
0: \text { Nein }\end{array}$ \\
\hline
\end{tabular}


Mittelwert aus allen Unterkategorien berechnet (vgl. dazu Tab. 1). Zusätzlich zu den Points of Contact wurde auch die Gemeindegrösse erhoben, um den Einfluss der Grösse einer Gemeinde mit dem Angebot an Kontaktmöglichkeiten zu vergleichen. Dafür wurden alle Gemeinden gemäss ihrer Grösse in die folgenden Gruppen eingeteilt: ,0-2000 Einwohner*innen“, ,2001-5000 Einwohner*innen“, ,,5001-10.000 Einwohner*innen“, „10.001-20.000 Einwohner*innen“ und „Mehr als 20.000 Einwohner*innen“. Dieses Vorgehen ermöglicht eine Übersicht über das aktuelle Angebot von digitalen Points of Contact in den Zürcher Gemeinden.

\section{Ergebnisse}

Im Folgenden werden die Ergebnisse aus der Erhebung verschiedener Kontaktmöglichkeiten aller 162 Zürcher Gemeinden präsentiert. Dabei wird insbesondere auf die Unterschiede zwischen den Gruppen basierend auf der Grösse der Gemeinden eingegangen.

\subsection{Digitale Points of Contact}

Eine Übersicht zu den angebotenen digitalen Kontaktmöglichkeiten gibt Abb. 1. Es zeigt sich, dass nicht alle erhobenen Kontaktmöglichkeiten gleich häufig von den Gemeinden angeboten werden: Alle Gemeinden bieten mindestens eine E-MailAdresse (Allgemeine, Amt, Mitarbeitende) auf ihrer Webseite an. Insgesamt haben 93,2\% der 162 Gemeinden eine allgemeine E-Mail-Adresse auf der Webseite, 86,4\% diejenige der verschiedenen Ämter und 92,6\% geben auch die E-Mail-Adressen einzelner Mitarbeitenden auf der Webseite bekannt. Am zweithäufigsten bieten Gemeinden einen Online-Schalter an, nämlich 96,3\% der Zürcher Gemeinden. Auch ein Benutzer*innen- beziehungsweise Bürger*innenkonto wird von 55,6\% der Gemeinden angeboten. Die weiteren fünf erhobenen Points of Contact finden sich deut-

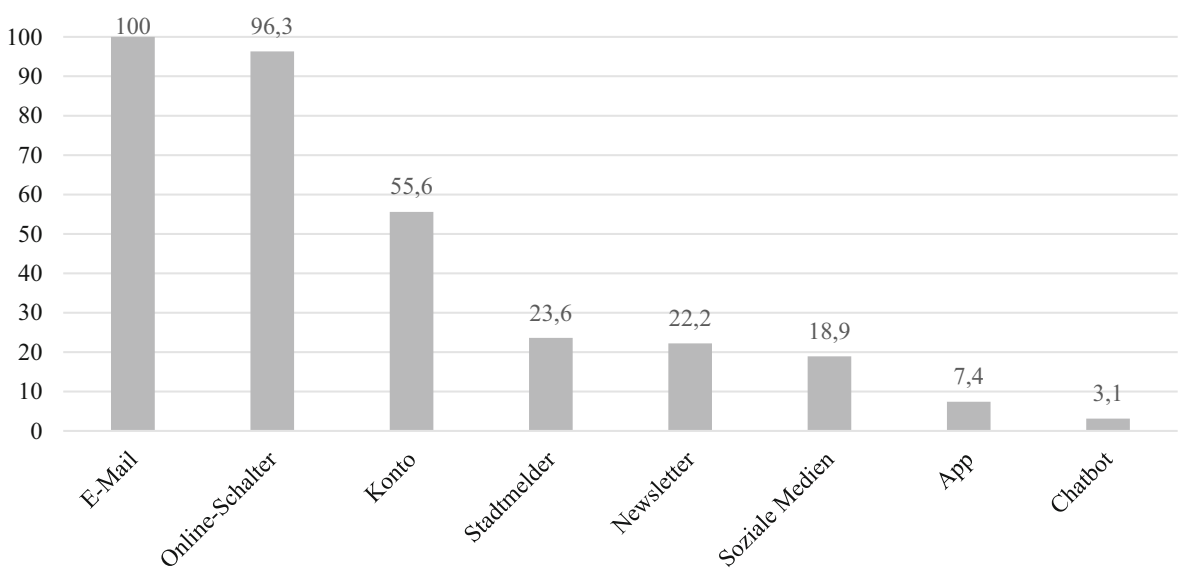

Abb. 1 Übersicht digitale Points of Contact. (Angaben in Prozent, $N=162$. Bei den zusammengesetzten Variablen „E-Mail“ und „Soziale Medien“ wird die Summe der Teilerhebungen ausgewiesen) 
lich weniger häufig auf den Gemeindewebseiten: Einen Stadtmelder führen 23,6\% der Gemeinden, einen Newsletter kann bei 22,2\% der Gemeinden abonniert werden und eine Verlinkung zu mindestens einem Sozialen Medium besteht bei 18,9\% der Gemeinden. Von diesen verwenden 15,4\% Facebook, 9,3\% Instagram, 8,6\% Twitter und 5\% YouTube. Am wenigsten häufig werden als digitale Points of Contact eine Gemeinde-App (7,4\%) sowie ein Chatbot $(3,1 \%)$ angeboten.

\subsection{Gemeindegrösse und digitale Points of Contact}

In einem zweiten Schritt wird nun das Angebot der digitalen Kontaktmöglichkeiten mit der Grösse einer Gemeinde verglichen. Dafür wurden die Gemeinden nach Grösse geordnet in fünf Gruppen unterteilt ${ }^{1}$ : Gruppe 1: 0-2000 Einwohner*innen, Gruppe 2: 2001-5000 Einwohner*innen, Gruppe 3: 5001-10.000 Einwohner*innen, Gruppe 4: 10.001-20.000 Einwohner*innen und Gruppe 5: Mehr als 20.000 Einwohner*innen. Das Minimum für den Gesamtscore liegt bei 0,125 und das Maximum bei 0,875. Im Mittel erreichen die Gemeinden einen Wert von 0,386 (SD=0,140). Aufgeteilt in die Gruppen nach Anzahl Einwohner*innen ergibt sich folgendes Bild: Die Gruppe mit 0-2000 Einwohner*innen erzielt eine mittlere Bewertung von 0,306 (SD=0,105, N=46), welche die tiefste Bewertung aller Gruppen darstellt. Die Gruppe mit 2001-5000 Einwohner*innen erreicht einen mittleren Score von 0,376 ( $\mathrm{SD}=0,118, N=45)$, die Gruppe mit 5001-10.000 Einwohner*innen eine mittlere Bewertung von 0,397 ( $\mathrm{SD}=0,117, N=39)$ und die Gruppe mit 10.001-20.000 Einwohner*innen erzielt im Schnitt einen Wert von 0,446 ( $\mathrm{SD}=0,147, N=21)$. Die höchsten Bewertungen erhalten Gemeinden der Gruppe mit mehr als 20.000 Einwohner*innen, die bei $0,609(\mathrm{SD}=0,132, N=11)$ liegt. Abb. 2 verdeutlicht diese Ergebnisse, welche einen positiven Zusammenhang zwischen der Anzahl Einwohner*innen und der Vielfalt an angebotenen Kontaktmöglichkeiten vermuten lassen. Eine Korrelationsanalyse nach Pearson zeigt, dass diese Vermutung zutreffen dürfte: Die Grösse einer Gemeinde korreliert positiv mit dem Gesamtscore $(r=0,508)$. Dieser Befund ist auf dem 1\%-Konfidenzniveau hochsignifikant $(p=0,001)$.

Um auf signifikante Unterschiede zwischen den Gruppen zu testen, wurde eine Varianzanalyse ${ }^{2}$ durchgeführt. Diese bestätigt die Vermutung, dass Gemeinden mit einer höheren Anzahl Einwohner*innen einen höheren Gesamtscore erreichen und damit ein vielfältigeres Angebot an digitalen Kontaktmöglichkeiten anbieten. Die Varianzanalyse zeigt signifikante Gruppenunterschiede auf dem 1\%-Konfidenzniveau $(\mathrm{F}=19$ '216, $p=0,001, N=160)$. Die Effektstärke $\omega^{2}$ liegt bei 0,313 und zeugt somit von einem starken Effekt. Der Tukey-Kramer Post-hoc Test zeigt zudem, dass sich der mittlere Gesamtscore der Gruppe der kleinsten Gemeinden signifikant negativ von den grösseren Gruppen 3 bis 5, nicht aber von der kleine-

\footnotetext{
1 Die Einteilung in die Gruppen wurde vom Verein Züricher Gemeindeschreiber und Verwaltungsfachleute übernommen (VZGV 2020).

2 Für die Varianzanalyse wurden extreme Ausreisser (Abstand ist grösser als 3-mal den Interquartilsabstand zum Mittelwert, in Abb. 2 mit * markiert) ausgeschlossen (Gemeinden Höri und Niederweningen der Gruppe 2). Für die Überprüfung der Varianzhomogenität wurde der Levene-Test angewendet, welcher Homoskedastizität in den Daten bestätigt $(p=0,088)$.
} 


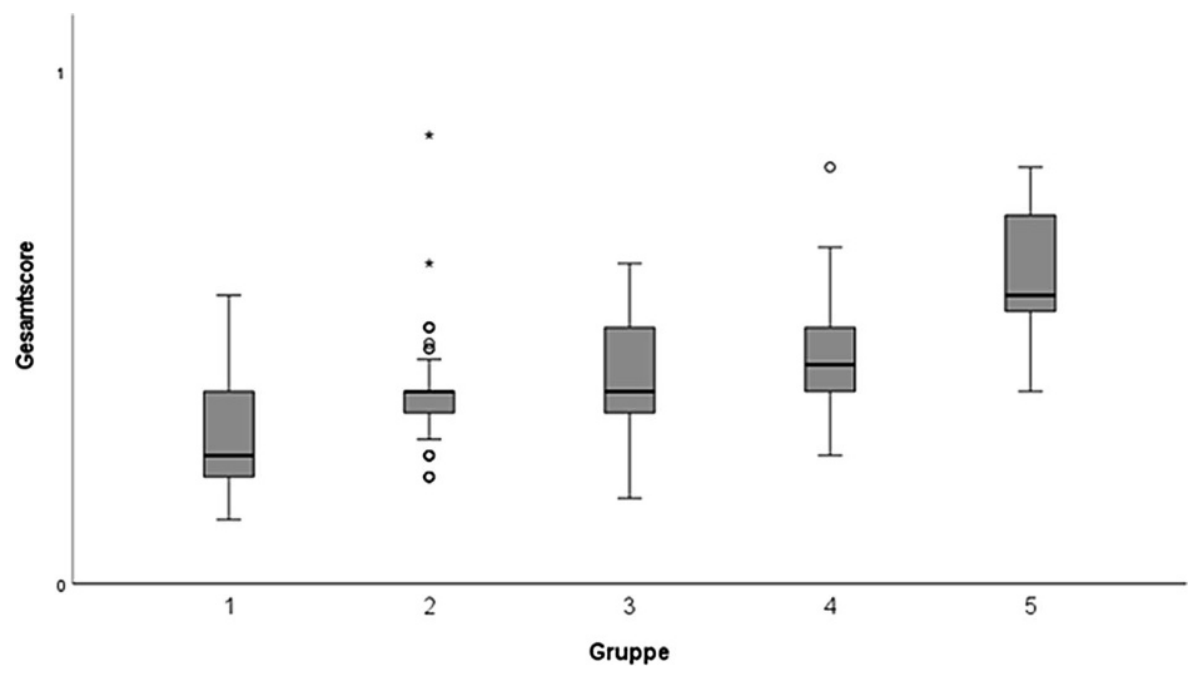

Abb. 2 Boxplot zu Gemeindegrösse und Gesamtscore. ( $N=162$. Gruppe 1: 0-2000 Einwohner*innen, Gruppe 2: 2001-5000 Einwohner*innen, Gruppe 3: 5001-10.000 Einwohner*innen, Gruppe 4: 10.00120.000 Einwohner*innen, Gruppe 5: Mehr als 20.000 Einwohner*innen. Boxplot mit Minimum, Mittelwert, Maximum und Ausreisser)

ren Gruppe 2 unterscheidet: Die Mittelwertdifferenz zu Gruppe 2 beträgt $-0,052$ ( $\mathrm{SE}=0,0234, p=0,178)$, zu Gruppe $3-0,091$ ( $\mathrm{SE}=0,024, p=0,002)$, zu Gruppe 4 $-0,140$ ( $\mathrm{SE}=0,029, p=0,001)$ und zu Gruppe $5-0,303$ (SE=0,024, $p=0,001$ ). Somit bieten kleinere Gemeinde signifikant weniger digitale Kontaktmöglichkeiten an als grössere Gemeinde und die Hypothese 1 kann vorläufig angenommen werden.

\section{Schlussbetrachtungen}

Dieser Beitrag untersucht das Angebot an digitalen Points of Contact von Schweizer Gemeinden am Fallbeispiel des Kanton Zürichs. Um die Forschungsfrage Welche digitalen Kontaktmöglichkeiten bestehen in den Zürcher Gemeinden und inwiefern hängt das Angebot mit der Grösse der Gemeinde zusammen? zu beantworten, wurden digitale Kontaktmöglichkeiten aller 162 Zürcher Gemeinden über deren Webseiten erhoben. Es wurde vermutet, dass das Angebot bei grösseren Gemeinden vielfältiger ausfällt, da mehr Ressourcen vorhanden sind.

Die Ergebnisse zeigen, dass die digitalen Kontaktmöglichkeiten „E-Mail“ und „Online-Schalter“ von den Gemeinden am häufigsten angeboten werden. Diese werden von fast allen der 162 Gemeinden auf der Webseite angegeben. Weniger häufig sind Kontaktmöglichkeiten, die in den letzten Jahren aufgekommen sind, wie ein Stadtmelder oder ein Chatbot. Auch die Sozialen Medien werden von einer Mehrheit der Gemeinden heute nicht zur Kommunikation mit der Bevölkerung verwendet. Des Weiteren zeigen die Ergebnisse signifikante Unterschiede zwischen kleinen und grossen Gemeinden: Grössere Gemeinden haben ein vielfältigeres Angebot an digitalen Kontaktmöglichkeiten als kleinere Gemeinden, was mit den verfügbaren 
Ressourcen erklärt werden dürfte. Insgesamt zeigt sich aber über alle Gemeinden, dass das Potenzial von digitalen Points of Contact, das in der Privatwirtschaft bereits anerkannt wird, noch nicht ausgeschöpft wird. Dementsprechend können die Zürcher Gemeinden heute auch noch nicht vollumfänglich von den verschiedenen Vorteilen wie Effizienzsteigerung, Automatisierungspotentiale, die digitalen Points of Contact nachgesagt werden, profitieren.

Aus den präsentierten Ergebnissen lässt sich schliessen, dass die Gemeinden noch wenig Gebrauch von innovativen Points of Contact wie einem Chatbot oder Stadtmelder machen. Diese Aussage wird auch dahingehend unterstützt, dass weitere Kontaktmöglichkeiten wie beispielweise die Partizipationsplattform Crossiety aufgrund ungenügender Beobachtungen als Points of Contact für die vorliegende Analyse ausgeschlossen wurden. Dieser Umstand könnte mit dem fehlenden Leistungsdruck, den Kund*innen und Unternehmen auf die Verwaltung ausüben sowie auch der Tatsache, dass die Schweizer Verwaltungen bezüglich der Digitalisierung im Vergleich zu anderen Ländern eher zurückhaltenden zeigen, erklärt werden (vgl. dazu Engels et al. 2018).

Die Ergebnisse müssen insofern mit Vorsicht interpretiert werden, da sie nur eine Aussage für Zürcher Gemeinden erlauben. Inwiefern sich diese auf andere öffentliche Verwaltungen übertragen lassen, bleibt offen. Zudem kann argumentiert werden, dass die Gemeindegrösse als Platzhalter für die Ressourcen unzureichend beziehungsweise zu ungenau ist. Die Grösse der Gemeinde als erklärender Faktor für die Vielfältigkeit des Angebots an digitalen Points of Contact ist somit nur als eine Annäherung zu verstehen.

Insgesamt versteht sich dieser Beitrag als eine erste Übersicht zu den digitalen Kontaktmöglichkeiten in Zürcher Gemeinden, auf dessen Ergebnissen mit weiteren Studien aufgebaut werden kann. Um die Befunde zu vertiefen, wären Studien zur Nutzung der digitalen Kontaktmöglichkeiten sowie eine Bedarfsanalyse auf der Seite der Nutzenden aufschlussreich. Daraus könnten Handlungsempfehlungen abgeleitet werden, in welche Richtung sich das Angebot von digitalen Kontaktmöglichkeiten der Gemeinden entwickeln sollte. Zudem sollten auch die Ursachen des unterschiedlichen Angebotes verstärkt untersucht werden, da auch weitere Faktoren als nur die Gemeindegrösse einen Einfluss darauf haben könnten, wie beispielsweise das interne Know-how. Mit diesen Erkenntnissen könnten Möglichkeiten für kleinere Gemeinden erarbeitet werden, die fehlenden Faktoren zu kompensieren. Ferner sollte auch die Rolle der zunehmenden Anzahl Start-Ups im Bereich der Digitalisierung von Verwaltungen (bspw. Crossiety, E-Mitwirkung) auf die digitale Transformation der Gemeinden in Folgeuntersuchungen genauer betrachtet werden.

Zusammenfassend lässt sich festhalten, dass der Aufbau eines E-Governments in den untersuchten Gemeinden noch nicht abgeschlossen ist. Während Gemeinden im Bereich der digitalen Points of Contact eher auf die Klassiker wie E-Mail oder Online-Schalter setzten, werden innovative Kontaktmöglichkeiten wie ein Stadtmelder oder ein Chatbot seltener angeboten. Die Grösse einer Gemeinde scheint zudem einen Einfluss auf die Vielfalt des Angebotes an digitalen Points of Contact zu haben. Es bedarf weiterer Forschung, um die Ursachen genauer zu beleuchten und konkrete Handlungsempfehlungen für Gemeinden abzuleiten, die den Weg der digitalen Transformation noch weiter gehen wollen. 
Funding Open access funding provided by ZHAW Zurich University of Applied Sciences

Open Access Dieser Artikel wird unter der Creative Commons Namensnennung 4.0 International Lizenz veröffentlicht, welche die Nutzung, Vervielfältigung, Bearbeitung, Verbreitung und Wiedergabe in jeglichem Medium und Format erlaubt, sofern Sie den/die ursprünglichen Autor(en) und die Quelle ordnungsgemäß nennen, einen Link zur Creative Commons Lizenz beifügen und angeben, ob Änderungen vorgenommen wurden.

Die in diesem Artikel enthaltenen Bilder und sonstiges Drittmaterial unterliegen ebenfalls der genannten Creative Commons Lizenz, sofern sich aus der Abbildungslegende nichts anderes ergibt. Sofern das betreffende Material nicht unter der genannten Creative Commons Lizenz steht und die betreffende Handlung nicht nach gesetzlichen Vorschriften erlaubt ist, ist für die oben aufgeführten Weiterverwendungen des Materials die Einwilligung des jeweiligen Rechteinhabers einzuholen.

Weitere Details zur Lizenz entnehmen Sie bitte der Lizenzinformation auf http://creativecommons.org/ licenses/by/4.0/deed.de.

\section{Literatur}

Baller S, Dutta S, Lavin B (2016) The global information technology report 2016. Innovating in the digital economy. World economic forum. http://www3.weforum.org/docs/GITR2016/WEF_GITR_Full_ Report.pdf. Zugegriffen: 11. März 2021

Brüesch C, Mertes A, Fischer D, Giger M-A, Lamprecht M (2020) Stimmen von Schweizer Grossunternehmen zur digitalen Verwaltung der Schweiz. ZHAW School of Management and Law/KPMG, S 26

Brüesch C, Mertes A, Flick Witzig M, Giger M-A, Steinbrecher M (2017) Digitale Verwaltung Eine Studie des Institutes für Verwaltungs-Management (IVM) und KPMG Schweiz. ZHAW School of Management and Law

Buess M, Ramsden A, Bieri O (2019) Nationale E-Government-Studie 2019 - Www.egovernment.ch. Staatssekretariat für Wirtschaft (SECO). https://www.egovernment.ch/de/dokumentation/nationalee-government-studie-2019/. Zugegriffen: 11. März 2021

Cahlikova T, Bundi P (2020) Managing the rise of the digital state: implementation of digital education by local government. Swiss Yearb Adm Sci 11(1):145-157. https://doi.org/10.5334/ssas. 144

C-Factor (2019) Gemeinde-Kommunikation. https://www.cfactor.ch/blog/blog-gemeinde-kommunikation/. Zugegriffen: 11. März 2021

Dahl AJ, D'Alessandro AM, Peltier JW, Swan EL (2018) Differential effects of omni-channel touchpoints and digital behaviors on digital natives' social cause engagement. J Res Interact Mark 12(3):258-273. https://doi.org/10.1108/JRIM-04-2018-0051

Engels B, Wallner C, Ammann M, Schnell F (2018) „Max Weber in der Digitalisierungsfalle?“ Avenir Suisse. https://www.avenir-suisse.ch/publication/max-weber-in-der-digitalisierungsfalle/. Zugegriffen: 13. Jan. 2021

Dietrich S, Stoll A, Brüesch C (2018) Wo ein Wille ist, ist auch ein Weg - Einflussfaktoren für die Entwicklung von Smart Government in der Schweiz auf subnationaler Ebene. Jahrbuch Der Schweizerischen Verwaltungswissenschaften 9(1):80-95. https://doi.org/10.5334/ssas.121

European Commission Joint Research Centre (2020) A multi-dimensional framework to evaluate the innovation potential of digital public services: A step towards building an innovative public services observatory in the EU. Publications Office. https://data.europa.eu/doi/10.2760/09628. Zugegriffen: 11. März 2021

E-Government Schweiz (2019) E-GovernmentStrategie Schweiz 2020-2023. Geschäftsstelle E-Government Schweiz. https://www.egovernment.ch/de/umsetzung/e-government-strategie/. Zugegriffen: 11. März 2021

Fischer D, Brändle F, Mertes A, Pleger LE, Rhyner A, Wulf B (2020a) Partizipation im digitalen Staat: Möglichkeiten und Bedeutung digitaler und analoger Partizipationsinstrumente im Vergleich. Swiss Yearb Adm Sci 11(1):129-144. https://doi.org/10.5334/ssas.141 
Fischer S, Lux A, Guerrero R, Ahmad R, Lohrenz L, Lattemann C (2020b) Digitalisierung als Grundlage wertvoller Zusammenarbeit - Die Gestaltung von Service-Ökosystemen in den personennahen Dienstleistungen. HMD 57(4):655-668. https://doi.org/10.1365/s40702-020-00640-9

Mertes A, Pleger LE, Bossert PB, Keller A, Schmidli FH (2020) Digitalisierungsfortschritt föderaler Einheiten: Ein Vergleich der Ausgestaltungen kantonaler ePlattformen in der Schweiz. Swiss Yearb Adm Sci 11(1):38-54. https://doi.org/10.5334/ssas.132

Neuroni A, Kissling-Näf I, Riedl R (2019) E-Government und smarter Staat: Die Schweiz auf halbem Weg. In: Stember J, Eixelsberger W, Spichiger A, Neuroni A, Habbel F-R, Wundara M (Hrsg) Handbuch E-Government: Technikinduzierte Verwaltungsentwicklung. Springer, Wiesbaden, S 163-180. https://doi.org/10.1007/978-3-658-21402-9

Pleger LE, Mertes A, Rey A, Brüesch C (2020) Allowing users to pick and choose: a conjoint analysis of end-user preferences of public e-services. Gov Inf Q 37(4):101473. https://doi.org/10.1016/j.giq. 2020.101473

Schedler K, Proeller I (2011) New public management. Haupt, Bern, Stuttgart, Wien

Statista (2019) Internetnutzung in der Schweiz. Statista. https://de.statista.com/themen/2664/internetnutzungin-der-schweiz/. Zugegriffen: 11. März 2021

Straker K, Wrigley C, Rosemann M (2015) Typologies and touchpoints: designing multi-channel digital strategies. J Res Interact Mark 9(2):110-128. https://doi.org/10.1108/JRIM-06-2014-0039

Teucher M, Lang A (2020) Die Digitalisierung von Verwaltungsleistungen: Die Auswirkungen auf die Bearbeitungszeit am Beispiel des eUmzugCH. Swiss Yearb Adm Sci 11(1):94-107. https://doi.org/ $10.5334 /$ ssas. 142

United Nations (2020) E-Government Survey 2020. United Nations. Departement of Economic and Social Affairs

VZGV (2020) Auswertung Online Umfrage. https://www.vzgv.ch/digitalisierung. Zugegriffen: 11. März 2021

Weber M (1980) Wirtschaft und Gesellschaft. Mohr Siebeck, Tübingen

Wong W, Welch E (2004) Does E-government promote accountability? A comparative analysis of website openness and government accountability. Governance 17(2):275-297. https://doi.org/10.1111/j. 1468-0491.2004.00246.x 